\title{
La transposición del concepto de diversidad biológica. Un estudio sobre los libros de
} texto de la educación secundaria española Didactic transposition of the biodiversity concept. A study
of Spanish high-school textbooks

Gonzalo M.A. Bermudez

Universidad Nacional de Córdoba, Facultad de Ciencias Exactas,

Físicas y Naturales, Argentina.

CONICET (Consejo Nacional de Investigaciones Cientificas y Técnicas), Argentina.

Universidad de Valencia, Facultad de Magisterio, Departamento de Didáctica

de las Ciencias Experimentales y Sociales

gbermudez@com.uncor.edu

Sandra Díaz

Universidad Nacional de Córdoba,

Facultad de Ciencias Exactas, Físicas y Naturales, Argentina.

CONICET (Consejo Nacional de Investigaciones Cientificas y Técnicas), Argentina.
Ana Lía De Longhi

Universidad Nacional De Córdoba, Facultad de

Ciencias Exactas, Físicas y Naturales, Argentina.

\author{
Valentín Gavidia Catalán \\ Universidad de Valencia, Facultad de Magisterio, \\ Departamento de Didáctica de las Ciencias \\ Experimentales y Sociales.
}

RESUMEN • Este trabajo estudia la presencia y complejidad en el tratamiento del concepto de diversidad biológica en libros de texto de educación secundaria, tanto de la obligatoria (ESO) como del bachillerato de España. Se recopilaron trabajos científicos de la «comunidad de expertos» con el objetivo de definir el ámbito conceptual de la biodiversidad, que actúa de vigilante epistemológico en el proceso de transposición didáctica. Los resultados mostraron una multiplicidad de definiciones de la biodiversidad en libros de texto, con un fuerte predominio de reducción a la idea de número de especies (riqueza), lo que pone en evidencia su desactualización. A la luz de este estudio, el listado de contenidos del currículo oficial no conduce a una transposición de una conceptualización de biodiversidad más coherente con la comunidad de expertos. Según los niveles de complejidad encontrados, recomendamos el uso de libros de la materia «Ciencias de la tierra y medioambientales» de segundo curso del bachillerato para estudiar la biodiversidad.

PALABRAS CLAVE: diversidad biológica; manuales escolares; especies; transposición didáctica; vigilante epistemológico.

ABSTRACT - This paper studies the existence and complexity of the treatment of the biodiversity concept in middle and high school Spanish textbooks. Scientific papers from the «expert community» were reviewed in order to define the biodiversity conceptual framework, which acts as the epistemological vigilance during the process of didactic transposition. This revealed a multiplicity of biodiversity definitions, with a strong predominance of the number of species (richness), suggesting that many textbooks were outdated. In the light of the present study, the official curriculum does not lead to the transposition of a biodiversity conceptualization which is more in line with that of the expert community. Regarding the levels of complexity, we recommend the use of the "earth and environmental sciences» textbooks of the last year of high school in order to study biodiversity.

KEYWORDS: biological diversity; textbooks; species; didactic transposition; epistemological vigilance.

Fecha de recepción: marzo 2013 • Aceptado: octubre 2013

Bermudez, G., De Longhi, A., Díaz, S., Gavidia, V. (2014) La transposición del concepto de diversidad biológica. Un estudio sobre los libros de texto de la educación secundaria española. Enseñanza de las Ciencias, 32.3, pp. 285-302 


\section{INTRODUCCIÓN}

Las instancias de enseñanza y aprendizaje de las ciencias, como de otras disciplinas, están mediadas por las concepciones alternativas de los estudiantes, las cuales prevalecen, se superponen e incluso construyen en la educación formal (Pozo y Gómez Crespo, 1998). Además, como tantos otros contenidos de las ciencias naturales, los temas ecológicos suelen presentarse en la escuela como un conjunto de dogmas, sobre todo cuando los alumnos estudian el ecosistema o las relaciones ecológicas como conceptos cerrados, estáticos y con una única formulación posible (Barker y Slingsby, 1998; García, 2003). Los trabajos desarrollados en el ámbito de la enseñanza de la Ecología se centran frecuentemente en conocer el pensamiento de los estudiantes sobre la estructuración de las redes tróficas y las nociones de equilibrio ecosistémico y de población (Bermudez y De Longhi, 2008; Munson, 1994: Özkan et al., 2004; Sánchez Cañete y Pontes Pedrajas, 2010). En cuanto a la enseñanza y el aprendizaje de la biodiversidad, se han encontrado dificultades asociadas a:

a) la utilización de los términos biodiversidad y número de especies como sinónimos, dejando de lado otros componentes (genes, poblaciones, grupos funcionales, comunidades y unidades de paisaje) y atributos (abundancia relativa, rango, distribución espacial e interacciones) (Bermudez et al., 2012a; Bermudez y De Longhi, 2008; Menzel y Bögeholz, 2009);

b) la consideración de que la biodiversidad se encuentra principalmente en países empobrecidos económicamente de África y América Central (Menzel y Bögeholz, 2009);

c) la existencia de un pensamiento mágico que presupone la bondad y armonía de los elementos de la naturaleza por el mero hecho de ser naturales (Rohde, 1996), con lo que se dejarían de considerar efectos negativos generados con la introducción y expansión acelerada de especies exóticas;

d) el reconocimiento y la valoración de especies, principalmente animales y, en menor medida, vegetales de contextos cercanos de las personas, como el jardín de la vivienda y la plaza de la comunidad, generalmente ricos en especies exóticas (Ballouard et al., 2011; Bermudez et al., 2012b; Campos, 2012; Campos et al., 2012; Nates et al., 2010; Arias Toledo et al., 2010);

e) el foco principal en «animales amigables» como los grandes mamíferos, especialmente aquellos con apariencia, inteligencia o comportamientos similares a los humanos, y en "plantas atractivas», con flores grandes, coloridas y perfumadas, o con frutos comestibles y atractivos (Ballouard et al., 2011; Campos, 2012; Lindermann-Matthies, 2005; Nates et al., 2010, Snaddon et al., 2008);

f) la valoración de la conservación de la biodiversidad solamente asociada al tipo de bienes y servicios ecosistémicos directos, como la provisión de alimentos y medicinas (Menzel y Bögeholz, 2009), y

g) la familiaridad con los organismos, que incrementa con la edad y difiere para niños y niñas, ya que mientras los primeros pueden reconocer mayormente las especies del campo o comunidades nativas circundantes, las niñas lo hacen con las especies de su jardín (Campos et al., 2012).

En el ámbito de la Didáctica, García Gómez y Martínez Bernat (2010) sostienen que para la enseñanza de la biodiversidad se debería dotar al alumnado de una base de competencias teórico-prácticas para fundamentar y orientar la interpretación crítica y la toma de decisiones sobre la conservación. Sin embargo, estos autores advierten que los libros de texto tratan la temática de la diversidad biológica de manera descontextualizada del ámbito social, aun cuando la dimensión ética y política de la biodiversidad es consustancial a su carácter científico y social. En coincidencia con García Gómez y Martínez Bernat (2010), hemos iniciado esta investigación sobre el concepto de biodiversidad y su compleja construcción escolar. 


\section{La transposición didáctica y los libros de texto}

El sociólogo Michel Verret propuso en 1975 el término transposición didáctica, que fue retomado por Yves Chevallard en 1980 en el contexto de la didáctica de la matemática. Este último autor estudió cómo el conocimiento científico se transforma para llegar a las aulas de la enseñanza obligatoria a través del fenómeno de transposición, experimentando adecuaciones particulares que configuran cierta pérdida de la historia y del contexto de descubrimiento o invención. Para Chevallard (1991), el conjunto de pasos por el que un objeto de saber se transforma en objeto de enseñanza se denomina transposición didáctica, en sentido amplio (sensu lato), mientras que la transformación de un objeto que enseñar en objeto de enseñanza es en un sentido estricto (stricto sensu). Según este autor, desde la ocurrencia del objeto de saber hasta el conocimiento enseñado tienen lugar dos tipos de envejecimiento. Existe un envejecimiento biológico, dado por cierto grado de discontinuidad entre lo que la comunidad científica investiga y lo que produce, ya sea porque se mantienen conceptos falsos en la escuela, ya porque nuevas producciones de la comunidad científica no tienen su correlato en el currículo escolar. También existe un envejecimiento moral, que ocurre cuando el saber enseñado no está de acuerdo con lo que la sociedad en sentido amplio plantea como necesario. El espacio donde ocurre el desequilibrio que origina algún tipo de envejecimiento es denominado noosfera y está integrado por los representantes del sistema de enseñanza, miembros de asociaciones docentes, profesores, padres de alumnos y especialistas de la disciplina.

Resulta conveniente mencionar que los manuales escolares forman parte de este proceso de adaptación curricular, a veces definiendo objetos de enseñanza y sus estrategias de instrucción, y otras, menos frecuentes, el objeto que enseñar. Para Cobo Merino y Batanero (2004), si un primer nivel de transposición se establece en los currículos, documentos y programas oficiales, los libros de texto escolares constituirían el segundo peldaño del proceso transpositivo. Esta fuerte influencia de los manuales se debe a que constituyen una ayuda inestimable para el profesor en el trabajo diario del aula, ofreciendo una concepción legitimada del saber a enseñar. Al respecto, Del Carmen y Jiménez Aleixandre (2010) dan cuenta de que el libro de texto ha sido y continúa siendo el material curricular más utilizado para la enseñanza de las ciencias en todos los niveles educativos. Los estudios sobre los manuales escolares de Biología se han centrado en la forma en que se introducen contenidos conceptuales y sus posibles errores (Gándara Gómez et al., 2002; González Rodríguez et al., 2003). Para temas de ecología, Ibarra Murillo y Gil Quílez (2005) estudiaron las diferencias en los significados que hay entre la ciencia de los científicos y la de los libros de texto con respecto a la teoría de la sucesión ecológica. Estos autores encontraron que la transposición se realiza a través de una simplificación de la teoría desde el modelo holista o ecosistémico, lo que, junto a la ambigüedad o polisemia de muchas de las nociones empleadas para explicar la sucesión, da lugar a significados deterministas sobre los cambios ecológicos. Por otro lado, Gavidia Catalán y Cristerna (2000) estudiaron de qué manera los libros de texto de educación secundaria obligatoria (ESO) de España tratan problemáticas ambientales de "Ciencias de la Naturaleza» y hasta qué punto ofrecen una visión medioambiental en su desarrollo. De sus hallazgos se destaca que los libros de texto:

a) no contemplan de forma adecuada los conceptos relacionados con el entorno local y más próximo del estudiante;

b) dan ejemplos de especies exóticas, como el tigre, el elefante y las secuoyas, pero rara vez de especies nativas;

c) no desarrollan la vertiente social de la problemática medioambiental (enfoque reduccionista), e

d) ilustran ecosistemas idealizados en los que el ser humano se encuentra ausente, salvo como agente de destrucción. 
En el presente estudio se describe cómo y en qué medida lo que forma parte del «saber experto» sobre la diversidad biológica se transpone en los libros de texto de la ESO y del bachillerato español.

\section{OBJETIVOS}

Elaborar una parrilla de análisis del ámbito conceptual de la biodiversidad que sea factible de ser aplicada a manuales escolares de educación secundaria.

Analizar la transposición didáctica del concepto de biodiversidad que se promueve a partir de los textos del bachillerato y de educación secundaria obligatoria.

Establecer niveles de complejidad en el tratamiento de los temas de biodiversidad en los libros de texto de educación secundaria obligatoria y del bachillerato, tras definir el conjunto de variables de análisis más importantes en la determinación de la variabilidad de los datos.

\section{METODOLOGÍA}

En una primera fase se determinó el ámbito conceptual de la biodiversidad definido por la comunidad de expertos a partir de la lectura de literatura especializada: revistas internacionales (artículos de investigación, revisiones, opiniones y ensayos) y enciclopedias destinadas al ámbito científico. Para ello se realizaron búsquedas sistemáticas en la base de datos bibliográficos Scopus, utilizando los términos biodiversity (biodiversidad) y biological diversity (diversidad biológica) como criterio de búsqueda en el resumen, título y palabras clave de artículos científicos publicados en revistas y enciclopedias internacionales desde el año 2000. El listado de trabajos fue ordenado por relevancia (opción dada por la plataforma Scopus) y los primeros veinte artículos se examinaron para identificar la conceptualización de la diversidad biológica. Las diferentes definiciones fueron codificadas para construir las categorías y subcategorías de análisis del presente estudio. Por razones de espacio, dichas categorías solo se presentan en la sección de resultados (tabla 1). A su vez, se realizaron cuatro entrevistas semiestructuradas a ecólogos con distintos objetos de estudio para entender las conceptualizaciones y formas de estudio de la diversidad biológica. Esta instancia de la investigación permitió revisar las categorías de análisis de los manuales escolares y ampliar las relaciones semánticas de la biodiversidad con otros conceptos ecológicos. Valiéndonos del tratamiento conceptual que se da en la «comunidad de expertos», el presente artículo se centra en responder desde qué perspectivas es posible analizar el tratamiento del concepto de biodiversidad en libros de texto de educación secundaria.

En una segunda etapa analizamos el programa nacional de estudios propuestos por el Ministerio de Educación de España, con el fin de determinar en qué espacios curriculares del bachillerato y ESO tiene presencia algún aspecto de la biodiversidad.

Por último, la tercera fase de este estudio comprendió el análisis de la transposición propiamente dicho, analizando la presencia del contenido en libros de texto de la ESO y del bachillerato. En esta etapa se seleccionaron los libros de texto de los últimos años (no anteriores a 1995), se registró la presencia/ ausencia de cada ítem de la parrilla de análisis y se transcribieron textualmente las citas, para finalizar con la expresión de resultados y la elaboración de conclusiones. En el análisis de los manuales se consideraron los capítulos dedicados a los temas «biodiversidad» $\mathrm{y}$ "problemáticas ambientales», según las prescripciones del currículo oficial. Se emplearon libros de texto de numerosas editoriales, incluidos materiales provenientes de la Comunitat Valenciana. A pesar de que no todas las editoriales están representadas, se considera que las analizadas constituyen una muestra suficientemente representativa de los libros de texto más utilizados durante las dos últimas reformas curriculares. El listado completo de libros ( $\mathrm{n}=60$, anexo I) recibió un código dado por su nivel educativo: «E» para la ESO ( $\mathrm{n}=27)$ y «B» 
para el bachillerato $(n=33)$. A su vez, se clasificaron por curso, es decir, «1» $(n=13)$ para manuales de primero de la ESO («Ciencias de la Naturaleza»), y «4» $(\mathrm{n}=14)$ para libros de cuarto de la ESO («Biología y Geología»). Para manuales del bachillerato, la codificación fue de «1» $(n=13)$ para libros de "Biología I», "Ciencias de la Naturaleza y de la Salud» y de "Biología y Geología», y de "2» (n = 20) para libros de «Biología II» $(\mathrm{n}=10)$ y «Ciencias de la Tierra y Medioambientales» $(\mathrm{n}=10)$. La variable respuesta fue la presencia o ausencia del tratamiento del contenido especificado en cada categoría de análisis. Debido a que no se encontró respuesta positiva en el análisis de los libros de «Biología II», estos fueron eliminados de los resultados.

El tratamiento estadístico de los datos se realizó mediante la prueba de Chi-cuadrado (Balzarini et al., 2008) y con dos métodos estadísticos multivariados, tales como el análisis de conglomerados (o cluster) y el análisis de componentes principales (ACP). El agrupamiento de objetos multivariados (conglomerados) es frecuentemente utilizado como método exploratorio de datos con la finalidad de obtener mayor conocimiento sobre la estructura de las observaciones y/o variables en estudio. A su vez, el ACP trata de encontrar, con pérdida mínima de información, un nuevo conjunto de variables (componentes principales), no correlacionadas entre sí, que expliquen la estructura de variación entre los casos observados (Balzarini et al., 2008). El sistema de coordenadas que explica la mayor varianza del conjunto de datos se denomina Componente 1, mientras que la segunda coordenada, Componente 2. La proporción de variabilidad total explicada por estas componentes (o autovalor) se expresa como porcentaje, logrando reducir la dimensionalidad de los datos a dos (Comp. 1 y Comp. 2) o más ejes cartesianos. Cada variable considerada en el ACP (en este caso, las categorías de análisis de los componentes y atributos de la biodiversidad) recibe un autovector, que muestra el coeficiente con que cada variable original es ponderada para conformar las componentes (Balzarini et al., 2008). En este sentido, las cifras de los ejes son relativas al análisis del conjunto de datos usados, no siendo comparables con ACP de otros datos. A su vez, mientras que las variables se grafican como vectores desde el origen (intersección entre Comp. 1 y Comp. 2), las observaciones (manuales escolares) son representados como puntos. Conviene destacar que en el ACP la distancia entre las observaciones y las variables no posee interpretación alguna, a diferencia de las direcciones de estos desde el origen. Es decir, los ángulos entre los vectores pueden ser interpretados en términos de las correlaciones entre variables; por ejemplo, ángulos de $90^{\circ}$ entre dos variables indican que ambas carecen de correlación (Balzarini et al., 2008). Los análisis se realizaron con los programas estadísticos SPSS (Statistical Package for Social Sciences, versión 17.0) e Infostat (Universidad Nacional de Córdoba, versión 2012).

\section{RESULTADOS Y DISCUSIÓN}

\section{A. El «saber experto»}

Sobre la base de la recopilación de bibliografía científica y entrevistas a expertos encontramos que el concepto de diversidad biológica ha sido heterogéneo desde al menos la primera mitad del siglo XX (Hamilton, 2005; Ghilarov, 1996). Con el paso del tiempo, la conceptualización de la biodiversidad se ha hecho más abarcadora, por ejemplo, en la definición de la Convención sobre la Diversidad Biológica, esta se define como la constituida por todos los organismos terrestres y acuáticos (incluyendo los animales, las plantas y los microbios) a todas las escalas, es decir, desde la diversidad genética dentro de las poblaciones, a la diversidad de especies, a la diversidad de comunidades a lo largo de los paisajes (CBD, 2001). A su vez, hallamos otras definiciones en artículos y enciclopedias científicas en las que se amplía el concepto, y la biodiversidad es entendida como la riqueza y composición de genotipos, especies, tipos funcionales y unidades de paisaje (Díaz, 2001a). En trabajos aún más recientes, por ejemplo el de Díaz et al. (2006), se mantienen las escalas de aplicación y se especifican aún más atributos, como 
la abundancia relativa, las interacciones y la distribución espacial de los componentes (genotipos, poblaciones, especies, etc.). A su vez, todos los ecólogos entrevistados señalaron el carácter polisémico del término biodiversidad y destacaron que «aun cuando sea frecuentemente entendido como la riqueza de especies, las definiciones más actuales superan ampliamente esta idea».

Es importante destacar que, en los últimos tiempos, la diversidad funcional ha emergido en la literatura ecológica como un aspecto de crucial importancia en la determinación de los procesos y servicios ecosistémicos (Tilman, 2001; Petchey y Gaston, 2006; Díaz et al., 2006 y 2007). En este sentido, existe un creciente consenso en que los efectos de la biodiversidad sobre los procesos ecosistémicos deberían ser atribuidos a los caracteres funcionales de las especies individuales y a sus interacciones más que el número de especies per se (Grime, 1997; Chapin et al., 2000; Díaz, 2001a y b). Ejemplos de caracteres funcionales son el peso y forma de las semillas, la composición química, textura y periodicidad de las hojas, el tamaño del organismo, la edad a la que comienza a reproducirse, etc. (Díaz y Cabido, 2001). El saber experto delineado en este apartado constituye la meta del proceso de construcción del conocimiento en el aula y actúa a modo de «vigilante epistemológico» (Chevallard, 1991) del tratamiento didáctico del concepto de biodiversidad que realizan los manuales escolares. Si bien en el presente artículo nos hemos centrado en los componentes y atributos de la biodiversidad, y en la coherencia interna de su uso en los libros de texto, en otros estudios hemos abordado las causas de la existencia, las estrategias de conservación y los servicios ecosistémicos que aporta la diversidad biológica.

\section{B. La presencia del concepto de biodiversidad en el currículo español}

\section{B.1. Educación secundaria obligatoria}

El conjunto de libros analizados en este estudio es coincidente con la vigencia de dos legislaciones educativas: la LOGSE (Real Decreto 1007/1991) y la LOE (Real Decreto 1631/2006). Sin embargo, tras un análisis comparativo, Banet (2007) señala que el nuevo currículo de la LOE propuso pocas novedades en cuanto a las finalidades y objetivos generales de la ESO, ya que tomó expresiones de la reforma anterior y desarrolló con distinto estilo y precisión solo algunos temas en particular (como la educación sexual). En este contexto, resulta conveniente destacar que debido a que los programas de estudio no incluyen el alcance de los términos que en él se expresan, se pone de manifiesto la libertad que tiene el profesorado para trabajar en los espacios libres del entramado curricular, adecuando las propuestas al proyecto institucional y a un enfoque de particular interés.

La organización de los espacios curriculares de la LOE ha determinado que, en el primer año, el concepto de biodiversidad sea tratado en la materia "Ciencias de la naturaleza", dentro del nuevo bloque 4, titulado «Los seres vivos y su diversidad» (MEC, 2006). Aquí se trabaja «la clasificación de los seres vivos en reinos (moneras, protoctistas, hongos, plantas y animales)» y «la valoración de la importancia de mantener la diversidad de los seres vivos. Análisis de los problemas asociados a su pérdida» (p. 694). En el segundo curso de la LOE, la biodiversidad se estudia indirectamente en el bloque 5, «La vida en acción», a través de los componentes de los ecosistemas y sus distintos tipos, reconociendo «el papel que desempeñan los organismos productores, consumidores y descomponedores en el ecosistema» (p. 695). Estos mismos contenidos ya eran tratados en la LOGSE en el bloque III, «La energía y los seres vivos». Por otro lado, en el bloque 6 del tercer curso de la LOE se tratan problemas ambientales relacionados con la contaminación del agua y del aire, y con los residuos y su gestión. A pesar de que en este se incluye la valoración del cuidado del medio ambiente y la adopción de conductas solidarias con él, no se menciona la pérdida de la biodiversidad como una problemática ambiental. En el currículo de la LOGSE, estos temas estaban ausentes, ya que en el tercer curso se estudiaba fundamentalmente anatomía y fisiología humana. 
Por último, en el cuarto curso de la ESO, en la materia "Biología y Geología», el bloque 3 determina el estudio de la «Valoración de la biodiversidad como resultado del proceso evolutivo. El papel de la humanidad en la extinción de las especies y sus causas» (MEC, 2006: 701). La concepción de biodiversidad asociada a la riqueza de especies subyace en este enfoque taxonómico y filogenético, que se promueve desde el primer curso. En Argentina hemos encontrado resultados similares en algunos lineamientos jurisdiccionales (Bermudez y De Longhi, 2012). En la reforma anterior se estudiaban los conceptos de especie, poblaciones y comunidades en el mismo bloque, tanto como los cambios naturales y antrópicos producidos en el ecosistema.

\section{B.2. Bachillerato}

En el bachillerato de la LOE (Real Decreto 1467/2007) la biodiversidad se trabaja tanto en materias comunes de las tres modalidades como en algunas específicas. Un caso paradigmático es el de la materia «Ciencias para el mundo contemporáneo» (MEC, 2007), ausente del programa que estableció la LOGSE (Real Decreto 1178/1992) (MEC, 1992). Este espacio curricular común surgió como respuesta a la demanda de que la biología debía formar parte de la cultura general y estar presente en todos los itinerarios del bachillerato (Cañal, 2008). En esta asignatura se estudia la biodiversidad en el bloque 2, «Nuestro lugar en el Universo», en relación con el origen de la vida, y en el bloque 4, «Hacia una gestión sostenible del planeta», estudiándose «La contaminación, la desertización. El aumento de residuos y la pérdida de biodiversidad...». Por otro lado, en la modalidad del bachillerato "Ciencias y Tecnología» de la LOE, en la materia del primer curso "Biología y Geología», se profundiza en los contenidos sobre taxonomía desarrollados en la ESO. Por ejemplo, el bloque 4, «Unidad y diversidad de la vida», incluye el contenido "La diversidad de los seres vivos y el problema de su clasificación. Criterios de clasificación» (MEC, 2007: 220). Asimismo, en los bloques 5 y 6 se analiza más detalladamente la diversidad de plantas y animales, lo que se corresponde con los temas 5, 7 y 8 de la LOGSE.

En el segundo curso del bachillerato de la LOE, la materia "Biología» (MEC, 2008) tiene una mirada netamente molecular y "pretende ofrecer una visión actualizada de la materia planteando la formación de los estudiantes en tres ámbitos [...], ampliar y profundizar los conocimientos científicos sobre los mecanismos básicos que rigen el mundo vivo, para lo cual es necesario tratar los niveles celular, subcelular y molecular, lo que permite explicar los fenómenos biológicos en términos bioquímicos o biofísicos» (p. 205). El enfoque de este espacio curricular pone en evidencia la idea de que los avances en biología solo se producen en las disciplinas genético-moleculares. $\mathrm{O}$, peor aún, que las explicaciones últimas sobre la naturaleza pueden ser reducidas exclusivamente a procesos moleculares. En este curso ni siquiera se menciona el componente genético de la biodiversidad al trabajar la «diversidad de microorganismos». De acuerdo con estas apreciaciones, Cañal (2008) afirma que los contenidos de Biología del bachillerato de la LOE se encuentran poco relacionados con el entorno inmediato y la biosfera. Las temáticas ambientales y la conceptualización de la diversidad biológica son recuperadas en la asignatura "Ciencias de la Tierra y Medioambientales» (CTM) del segundo curso de la LOE (MEC, 2008), la que se plantea como «una ciencia de síntesis y de aplicación de otras ciencias, entre las que figuran destacadamente la geología, la biología, la ecología, la química y la física, junto con otras aportaciones procedentes del campo de las ciencias sociales» (p. 226). El término biodiversidad figura en el bloque 4 de contenidos de CTM, al tratar «La biosfera como patrimonio y como recurso frágil y limitado. Impactos sobre la biosfera: deforestación y pérdida de la biodiversidad» (p. 230). En el caso del currículo de la LOGSE, estos conceptos se desarrollan en el tema 7, «La ecosfera: ecosfera, biosfera y ecosistema», con un tratamiento temático semejante. 


\section{Conceptualización de la biodiversidad y sus componentes en libros de texto}

La conceptualización de la diversidad biológica estuvo presente en la mayoría de los libros del bachillerato y de la ESO (tabla 1), sin existir diferencias significativas entre niveles educativos, aunque sí entre los cursos. El 100\% de los libros de segundo año del bachillerato definió la biodiversidad, mientras que solo lo hizo el 50 y el 38,5\% de los manuales de cuarto año de la ESO y de primero del bachillerato, respectivamente. Por un lado, esto muestra la gradualidad en el tratamiento del contenido y, por otro, que en la ESO pueden desarrollarse contenidos relacionados con la biodiversidad, aun sin que se presente una conceptualización de esta. Por ejemplo, para la ESO encontramos definiciones típicamente centradas en las especies: «¿Qué es la biodiversidad? Todos los organismos son semejantes en cuestiones fundamentales [...]. No obstante, hay características que diferencian a unos seres vivos de otros y los convierten en especies distintas. Denominamos biodiversidad a la gran variedad de especies distintas que constituyen la biosfera» [E1E] (p. 174), y «La diversidad actual de los seres vivos: se denomina diversidad biológica o biodiversidad a la variedad de seres vivos que habitan un lugar determinado. Hay diversas formas de calcular la biodiversidad. Una de las más sencillas es contar el número de especies que conviven en un territorio dado» [E4G] (p. 116).

Al comparar los niveles educativos hemos encontrado que la especie fue el componente de la biodiversidad más preponderante en los libros de texto (tabla 1). Los otros componentes, como el genético/ poblacional, comunitario/ecosistémico, paisajístico y de grupos funcionales estuvieron ausentes en la amplia mayoría de textos. Sin embargo, cabe destacar que las comunidades/ecosistemas, la trilogía de la biodiversidad y los grupos funcionales tuvieron un porcentaje de presencia significativamente más alto en el bachillerato que en la ESO. Por ejemplo, para el primer año del bachillerato encontramos una definición que incluye varios componentes no tenidos en cuenta en libros de la ESO:

Tabla 1.

Distribución de frecuencias relativas (en porcentaje) para las categorías de análisis correspondientes a la conceptualización de la diversidad biológica en libros de texto de la ESO y del bachillerato

\begin{tabular}{|c|c|c|c|c|c|c|c|c|c|}
\hline \multirow{3}{*}{ Categorías de análisis } & \multicolumn{3}{|c|}{ Nivel } & \multicolumn{5}{|c|}{ Curso } & \multirow{3}{*}{$\begin{array}{r}\text { Total } \\
\mathrm{n}=50\end{array}$} \\
\hline & ESO & BAC & \multirow{2}{*}{$X^{2}$} & ESO1 & ESO4 & BAC1 & BAC2 & \multirow{2}{*}{$X^{2}$} & \\
\hline & $\mathrm{n}=27$ & $\mathrm{n}=23$ & & $\mathrm{n}=13$ & $\mathrm{n}=14$ & $\mathrm{n}=13$ & $\mathrm{n}=10$ & & \\
\hline I. Presencia de conceptualización & 55,6 & 65,2 & ns & 61,5 & 50,0 & 38,5 & 100,0 & * & 60,0 \\
\hline 1. Genotipos/poblaciones & 11,1 & 39,1 & $*$ & 7,7 & 14,3 & 23,1 & 60,0 & $*$ a & 24,0 \\
\hline 2. Especies & 85,2 & 65,2 & ns & 84,6 & 85,7 & 38,5 & 100,0 & ** a & 76,0 \\
\hline 3. Comunidades/Ecosistemas & 14,8 & 39,1 & $*$ & 15,4 & 14,3 & 30,8 & 50,0 & $\mathrm{~ns}$ & 26,0 \\
\hline 4. Trilogía de la biodiversidad & 7,4 & 34,8 & $* \mathrm{a}, \mathrm{b}$ & 7,7 & 7,1 & 23,1 & 50,0 & $* \mathrm{a}$ & 20,0 \\
\hline 5. Paisajes & 3,7 & 8,7 & ns & 7,7 & 0,0 & 7,7 & 10,0 & ns & 6,0 \\
\hline 6. Grupos funcionales & 0,0 & 13,0 & ns & 0,0 & 0,0 & 15,4 & 10,0 & ns & 6,0 \\
\hline b) Inconsistencia & 74,1 & 21,7 & $* * *$ & 69,2 & 78,6 & 15,4 & 30,0 & $* * *$ a & 50,0 \\
\hline $\begin{array}{l}\text { c) Atributos } \\
1 . \text { Riqueza }\end{array}$ & 85,2 & 69,6 & ns & 84,6 & 85,7 & 46,2 & 100,0 & $* * a$ & 22,0 \\
\hline 2. Abundancia relativa & 14,8 & 34,8 & $\mathrm{~ns}$ & 0,0 & 28,6 & 0,0 & 80,0 & $* * * a$ & 24,0 \\
\hline 3. Composición & 0,0 & 4,3 & ns & 0,0 & 0,0 & 7,0 & 0,0 & ns & 2,0 \\
\hline 4. Rango & 0,0 & 0,0 & - & 0,0 & 0,0 & 0,0 & 0,0 & - & 0,0 \\
\hline 5. Interacciones & 14,8 & 8,7 & ns & 0,0 & 28,6 & 7,0 & 10,0 & ns & 12,0 \\
\hline 6. Distribución espacial & 3,7 & 0,0 & ns & 7,7 & 0,0 & 0,0 & 0,0 & ns & 2,0 \\
\hline
\end{tabular}

BAC = bachillerato; ESO = educación secundaria obligatoria; 1, 2 y 4 = ańos del nivel educativo correspondiente; $\mathrm{X}^{2}=$ valor de P de la prueba Chi-cuadrado de Pearson; * Significativo con $\mathrm{p} \leq 0,05$; $^{* *}$ significativo con $\mathrm{p} \leq 0,01$; ${ }^{* * *}$ significativo con

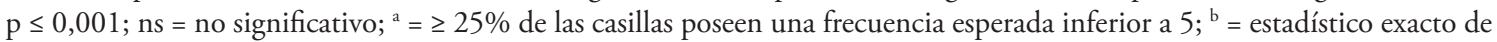
Irwin Fisher (significación bilateral) con $\mathrm{p} \leq 0,05$. 
En este libro [Elliot Norse y sus colaboradores] propusieron tres niveles de análisis para el estudio de la diversidad biológica: diversidad genética entre individuos de una especie dada; diversidad de especies, es decir, número de especies, y diversidad ecológica, variedad de comunidades en los ecosistemas. Por diversidad biológica se entiende la variabilidad de organismos vivos de cualquier fuente, incluidos, entre otros elementos, los ecosistemas terrestres y marinos, otros ecosistemas acuáticos y los complejos ecológicos de los que forman parte; comprende la diversidad dentro de cada especie, entre las especies y la de los ecosistemas [B1D] (p. 131).

Para segundo año del bachillerato hallamos una definición que, tras presentar la conceptualización de la CBD, se centra en los niveles genético, específico y ecosistémico:

De esta definición $[\mathrm{CDB}]$ se extraen los tres componentes de complejidad creciente de la diversidad biológica: la diversidad genética intraespecifica, la diversidad de especies y la diversidad de ecosistemas. Diversidad genética. Sin la gran variabilidad de genes que contienen las poblaciones, las especies serían incapaces de adaptarse al entorno y de evolucionar bajo la acción de la selección natural. Sin la diversidad de especies, las biocenosis se empobrecerían, se harían más vulnerables a las variaciones ambientales y se verían alterados los flujos de materia y energía en los ecosistemas y en la biosfera. Una reducción en la diversidad de ecosistemas, con la desaparición de ambientes (hábitats) que ello implicaría, repercutiría sobre la diversidad global de especies, ya que estas constituyen biocenosis ligadas a biotopos concretos [B2H] (p. 336).

Resulta conveniente aclarar que las diferencias significativas entre cursos para los componentes deben tomarse con cautela (salvo para genotipos/poblaciones), considerando solamente las relaciones cualitativas entre cursos. Esto se debe a que se encontraron frecuencias esperadas menores al límite teórico de 5 para más del $20 \%$ de los niveles del factor. Teniendo en cuenta estas consideraciones, la mayoría de los componentes de la biodiversidad estuvo representada en los manuales de segundo año del bachillerato (tabla 1). Además, solamente un libro de primero y otro de cuarto de la ESO definieron la biodiversidad por los genotipos, las especies y los ecosistemas («trilogía de la biodiversidad») (Hamilton, 2005). Estos resultados indican que el proceso transpositivo para la conceptualización de la diversidad biológica se ha atrasado en relación con las definiciones que se dan en la comunidad de científicos. Este fenómeno, descrito por Chevallard (1991) como «envejecimiento biológico», tiene lugar por un creciente distanciamiento entre lo que la comunidad de científicos sabe y el «saber a enseñar». Como propuesta didáctica ante este desequilibrio, García Gómez y Martínez Bernat (2010) han propuesto realizar un análisis de los significados atribuidos al concepto de biodiversidad en el ámbito político, científico y mediático.

Por otro lado, conviene mencionar que algunos libros fueron inconsistentes en la conceptualización de la biodiversidad debido a confusiones terminológicas en sus componentes, la presencia de definiciones contradictorias o al considerar la pérdida de un componente no explicitado previamente. Hemos encontrado este tipo de inconsistencias en el 70,4\% de los libros de la ESO frente a un 21,7\% de los libros del bachillerato (tabla 1). Por ejemplo, la conceptualización de la biodiversidad que presenta el libro E1A incluye el término que se define como una parte de la misma definición (circularidad o tautología): «El término biodiversidad incluye todas las especies existentes y también las diversas variedades de estas especies que habitan en el conjunto de los diversos ecosistemas, o biosfera» [E1A] (p. 137).

Otro caso es el referido al contexto didáctico en el cual se presentan las definiciones, ya que, por ejemplo, el libro E1G reduce la biodiversidad al número de especies tanto en el texto del capítulo de tratamiento específico (noveno) como en un glosario. Sin embargo, en el capítulo siguiente (décimo) se introduce una definición más correcta en una nota ampliatoria, sin retomar la conceptualización anterior. En libros de cuarto año de la ESO hallamos inconsistencias similares cuando se cae en definiciones circulares $(\mathrm{E} 4 \mathrm{~N})$ o incluso cuando, al tratar la conservación de la biodiversidad, se hace referencia a componentes de esta no descriptos con anterioridad: «La conservación del paisaje como 
estrategia de conservación de la fauna salvaje y todo el muestrario de hábitats naturales no se limitan exclusivamente a las especies que existen en la biósfera. La biodiversidad empieza en el nivel molecular, el genético, por lo que preservar la diversidad genética de los individuos de una población resulta fundamental...» [E4E] (p. 224).

Otro caso que queremos destacar es el del manual B1D, en el que se presentan tres definiciones diferentes de la biodiversidad: la trilogía de la diversidad, la conceptualización dada en la Convención sobre la Diversidad Biológica y el «conjunto de seres vivos que pueblan un ecosistema» (glosario).

\section{Atributos de la biodiversidad presentes en libros de texto}

En cuanto a los atributos de la diversidad biológica, la riqueza de especies fue el de mayor presencia en los manuales de ambos niveles educativos. En tanto, la frecuencia relativa y las interacciones se encontraron en porcentajes variables, entre un 8,4 y un $34,8 \%$ de los libros (tabla 1). La riqueza de especies fue ejemplificada en el texto E1C con el número de especies conocidas en el presente: «Actualmente se conocen alrededor de un millón y medio de especies, en sus dos terceras partes insectos. Sin embargo, recientes estudios demuestran que la mayor parte está por describir. [...]. Es decir, las especies conocidas podrían suponer tan solo un 5\% del total» (p. 183).

La «abundancia relativa» fue tratada en manuales de cuarto de la ESO en relación con cálculo de índices y con las sucesiones ecológicas: «Con el fin de obtener un valor cuantitativo de las poblaciones se realizan censos que permiten descubrir las regularidades en las relaciones entre los números de distintas especies. El número de especies y la abundancia relativa de las mismas proporciona información sobre su diversidad» [E4E] (p. 165), y «Un parámetro importante que crece mientras avanza la sucesión es la complejidad [...]. La diversidad ecológica es una variable que suele emplearse como medida de la complejidad y, por ende, de la madurez de un ecosistema. La diversidad ecológica crece con el número de especies y con el equilibrio numérico entre ellas» [E4E] (p. 196).

Las nociones de riqueza y abundancia relativa se complejizaron en el segundo curso del bachillerato, donde se proponen cálculos de índices de diversidad de especies: «Por ejemplo, un campo en el que se cultive una sola especie tendrá una $\mathrm{H}=0$, puesto que $\log _{2} 1=0$. En los arrecifes de coral se registran índices de biodiversidad elevados, con valores que oscilan entre 2,7 y 4,9» [B2D] (p. 93).

La idea de complejidad creciente en el currículo tiene su base en la de secuenciar contenidos como formas de transitar el aprendizaje, por ejemplo, de lo más simple a lo más complejo (García, 1997; Giordan y de Vecchi, 1988). Los resultados del presente trabajo coinciden con la propuesta de Bermudez y De Longhi (2006), quienes plantearon que antes de la enseñanza de la biodiversidad y el atributo de la frecuencia relativa se considera como eslabón previo el entendimiento de la biodiversidad como riqueza de especies. Conviene destacar que los restantes atributos de la biodiversidad (composición, rango, interacciones y distribución espacial) estuvieron casi completamente ausentes de los textos de ambos niveles educativos. 


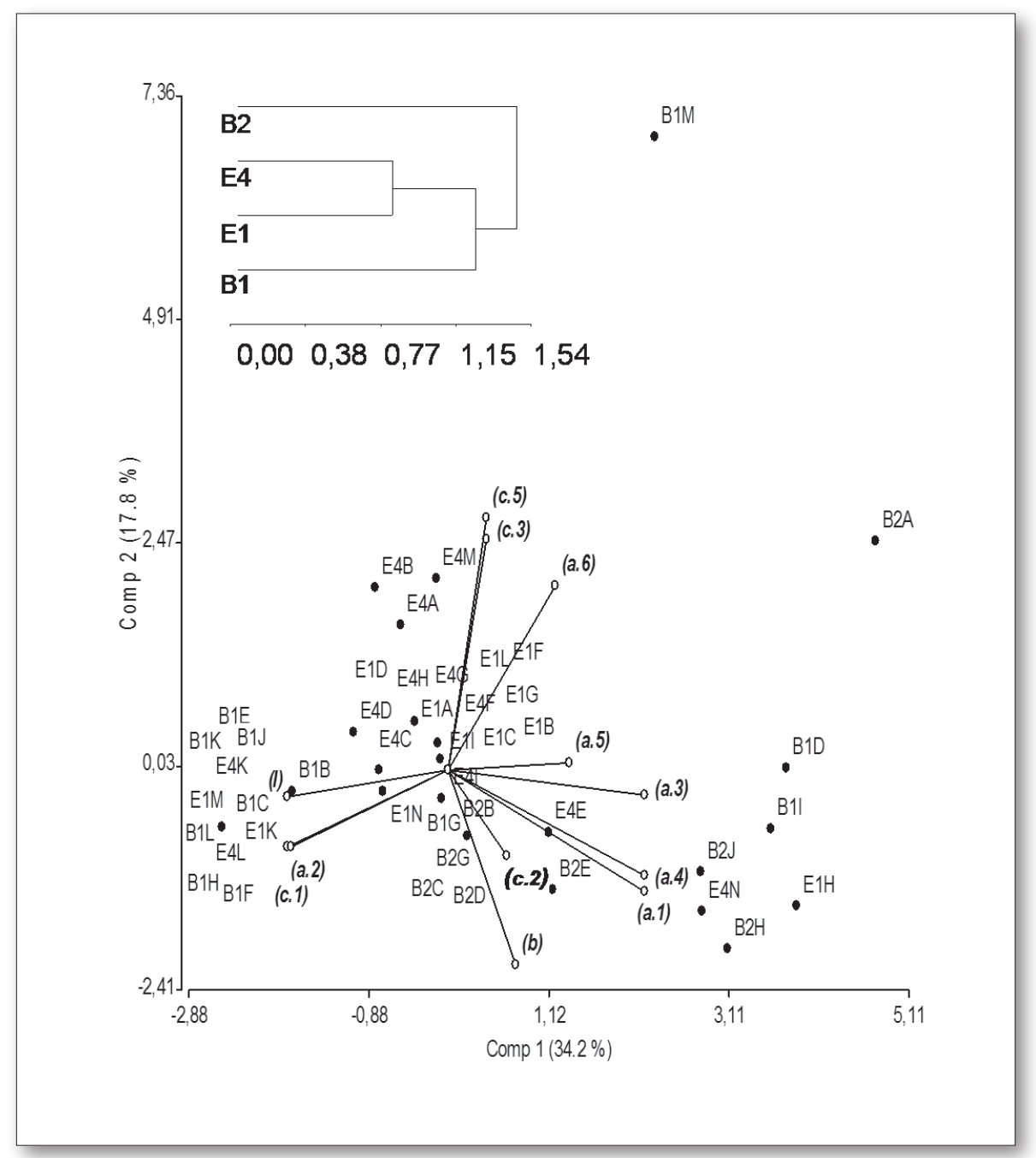

Fig. 1. Análisis de componentes principales (ACP) para el conjunto de variables y análisis de conglomerados (cluster) por ligamiento promedio y distancia euclídea (extremo superior izquierdo) para los libros de texto estudiados. Los porcentajes de varianza explicada se dan entre paréntesis. $\mathrm{E}=\mathrm{ESO} ; \mathrm{B}=$ bachillerato. Referencias: $\mathrm{I}$ = presencia de conceptualización; $\mathrm{a} .1$ = genotipos/poblaciones; a. 2 = especies; a. 3 = comunidades/ecosistemas; a.4 = trilogía de la biodiversidad; a. 5 = paisajes; a. 6 = grupos funcionales; b) inconsistencia; $\mathrm{c} .1$ = riqueza; $\mathrm{c} .2$ = abundancia relativa; $\mathrm{c} .3$ = composición; c. 5 = interacciones.

\section{E. Niveles de complejidad en el tratamiento de la biodiversidad en libros de texto}

Se realizó un análisis de componentes principales (ACP) para determinar cuáles son los factores (conjunto de variables) más importantes en la diferenciación de los libros de texto de la ESO y del bachillerato. Se encontró que las dos primeras componentes acumularon una proporción de varianza correspondiente al 52,0\%. La mayoría de las categorías de análisis sobre los componentes de la biodiversidad se distribuyeron en la componente 1 (34,2\%, figura 1). Este resultado indica que la mayoría de las relaciones conceptuales definidas en la primera etapa de la presente investigación tuvieron un peso importante para diferenciar los manuales estudiados. 
La figura 1 también muestra que componentes como los genotipos, las comunidades, los paisajes y la trilogía de la biodiversidad se encuentran inversamente relacionados con la presencia de la conceptualización, el componente especie y la riqueza. Estos resultados ponen en evidencia que cuando los libros de texto definen la biodiversidad, la mayoría lo hace como el número (riqueza) de especies. Además, la presencia de la conceptualización de la biodiversidad, la inclusión de componentes como las especies, las comunidades y paisajes y del atributo riqueza resultó independiente de la consistencia del texto (ángulos rectos entre estas variables e inconsistencia). Sin embargo, la inconsistencia aumentó junto a la trilogía de la biodiversidad, el componente genético y el atributo frecuencia relativa. Este resultado coincide con discusiones anteriores y se debe a que los manuales pueden ofrecer una definición de acuerdo con el «saber experto», pero reducirla al número de especies relacionarla con otros temas curriculares.

Se realizó además un análisis de conglomerados (cluster) (extremo superior izquierdo de la figura 1). Este análisis muestra que los textos de primer y cuarto curso de la ESO fueron los más similares entre sí, y luego estos con los manuales de primero del bachillerato. Estos resultados, junto con las pruebas Chi-cuadrado, indican que los textos de segundo curso del bachillerato de la materia «Ciencias de la Tierra y Medioambientales», fueron los más completos para tratar el concepto de diversidad biológica.

En relación con la diferenciación entre los libros de texto, los manuales se distribuyeron en tres zonas, las que pueden ser interpretadas como niveles de complejidad: a) la mayoría de los libros de segundo del bachillerato se ubicaron en el margen derecho de la figura 1, lo que representa el nivel más alto en el tratamiento conceptual de la biodiversidad; $b$ ) un nivel intermedio de complejidad quedó determinado por un conjunto de libros de cuarto y primero de la ESO, con algunos de segundo del bachillerato, y $c$ ) el nivel más bajo de complejidad estuvo integrado mayormente por libros de primero del bachillerato (margen izquierdo de la figura 1). Estos resultados muestran que no hubo grandes cambios en el nivel de complejidad del tratamiento conceptual de la biodiversidad de primero a cuarto curso de la ESO. Además, los manuales de primero del bachillerato representan el nivel más bajo de complejidad de educación secundaria. Por último, en función de estos resultados, recomendamos los libros ubicados en el margen derecho de la figura 1 para trabajar con los alumnos el tema de la diversidad biológica. En este sector de la figura encontramos manuales escolares de los cuatro cursos estudiados, por lo que su utilización garantizaría una adecuada transposición del concepto de biodiversidad en la educación secundaria española.

\section{CONSIDERACIONES FINALES}

En primer lugar, consideramos que la parrilla propuesta para el análisis del concepto de diversidad biológica, sus componentes y atributos permitió estudiar cuantitativa y cualitativamente la presencia de la conceptualización y las relaciones semánticas en libros de texto de la ESO y del bachillerato español. En este sentido, los resultados mostraron una multiplicidad de definiciones de la biodiversidad, con un claro predominio de la sinonimia con el número de especies (riqueza). Si bien estos resultados están de acuerdo con una cierta confusión terminológica en el ámbito científico (Hamilton, 2005), se evidencia que los libros de texto espańoles presentan definiciones desactualizadas de la diversidad biológica, tanto desde el punto de vista experto como político (Convención sobre la Diversidad Biológica). Este proceso de «envejecimiento biológico», en términos de la teoría de la transposición didáctica (Chevallard, 1991), alerta a la comunidad docente de la producción de materiales educativos y el uso de los libros de texto, tanto para el estudio de los estudiantes como para definir los alcances en el tratamiento del contenido. 
Según los altos porcentajes de inconsistencia en el tratamiento conceptual de la biodiversidad en manuales de la ESO y del bachillerato, creemos necesaria una actualización urgente y una adecuación externa e interna. Externa en cuanto a que deberían acortarse las distancias con las producciones de la "comunidad de expertos»; e interna en el sentido de lograr un grado superior de coherencia en el desarrollo temático. Sostenemos que incluir en la nueva edición de un manual escolar una definición más actual en un glosario o recuadro no basta para cubrir las expectativas de alfabetización científicatecnológica, el desarrollo de competencias específicas o la superación de visiones reduccionistas de la biodiversidad. Ampliar el ámbito de la noción de biodiversidad permitiría recuperar mayor cantidad de referentes cotidianos y generar una visión más crítica sobre el entorno. Además, en este elaborado proceso de transposición, el listado de contenidos presentes en los currículos oficiales no garantizaría que la conceptualización de la biodiversidad sea más coherente con la "comunidad de expertos».

En relación con la dimensión temporal en el tratamiento curricular, se evidenció una graduación en la complejidad del tratamiento de ciertos componentes y atributos. Por ejemplo, los conceptos de riqueza de especies se presentaron en los manuales de todos los cursos estudiados, mientras que los componentes ecosistémico y genético aparecieron solo en los de cuarto de la ESO. En un sentido similar, el atributo abundancia relativa fue registrado en los libros de texto de cuarto de la ESO y complejizado en segundo del bachillerato con el cálculo de índices de diversidad de especies. Estos resultados señalan la necesidad de profundizar en el análisis de las actividades para los alumnos en estudios futuros.

El análisis conjunto de todas las variables estudiadas nos permitió definir grupos de libros con diferentes tratamientos de la diversidad biológica. Por un lado, el grupo de libros con el tratamiento más complejo del tema fue explicado por los componentes genético/poblacional y comunitario/ecosistémico. En el nivel más bajo se encontraron los libros de primero del bachillerato y, luego, los de primero y cuarto de la ESO (que fueron los más similares entre sí). Por último, en el nivel más elevado de complejidad encontramos la mayoría de los textos de la materia "Ciencias de la Tierra y Medioambientales» del segundo curso del bachillerato.

Como recomendación para la práctica docente invitamos al profesorado a tomar decisiones sobre el diseño curricular, teniendo en cuenta los elementos del contexto de descubrimiento de la ciencia y proporcionando a los estudiantes una experiencia de aprendizaje significativa. Trabajar didácticamente sobre la selección y organización de un contenido como el de la biodiversidad exige una revisión disciplinar, una adecuada transposición y la elección de un enfoque que le otorgue a la propuesta de enseñanza un sentido ecológico y social. Para ello, el docente de Biología debe contemplar criterios lógicos, psicológicos y socioculturales al tomar decisiones sobre el conocimiento de enseñar. En este marco, resulta necesario establecer los niveles de complejidad del contenido prescripto desde los diseńos curriculares y los recursos didácticos necesarios. Lo anterior convierte al docente en un mediador calificado en la construcción del conocimiento y ejecutor de la trasposición, ya que es quien recupera el conocimiento cotidiano y lo proyecta hacia el científico desde una propuesta académica, legitimándolo en las interacciones áulicas que genera.

\section{AGRADECIMIENTOS}

A CONICET, Universidad Nacional de Córdoba, ANPCyT (FONCyT) y a los profesores del Departamento de Didáctica de las Ciencias Experimentales y Sociales de la Universitat de València.

\section{CONFLICTO DE INTERESES}

Los autores de este artículo declaran que no existe conflicto de intereses. 


\section{REFERENCIAS BIBLIOGRÁFICAS}

Arias Toledo, B.; Trillo, C. y Grilli, M. (2010). Uso de plantas medicinales en relación con el estado de conservación del bosque en Córdoba, Argentina. Ecología Austral, 20, pp. 235-246.

BANET, E. (2007). Nuevas enseñanzas mínimas para las ciencias de la naturaleza (biología y geología) en la ESO: ¿una reforma necesaria? Alambique. Didáctica de las Ciencias Experimentales, 53, pp. 77-94.

Ballouard, J.-M.; Brischoux, F. y Bonnet., X. (2011). Children prioritize virtual exotic biodiversity over local biodiversity. PLoS ONE, 6(8). e23152. doi:10.1371/journal.pone.0023152. http://dx.doi.org/10.1371/journal.pone.0023152

Balzarini, M.G.; González, L.; Tablada, M.; Casanoves, F.; Di Rienzo, J.A. y Robledo, C.W. (2008). Infostat. Manual del Usuario. Córdoba: Editorial Brujas.

Barker, S. y Slingsby, D. (1998). From nature table to niche: curriculum progression in ecological concepts. International Journal of Science Education, 20 (4), pp. 479-486.

http://dx.doi.org/10.1080/0950069980200407

Bermudez, G.M.A.; Batistón, L.V.; García Capocasa, M.C.; Díaz, S. y De Longhi, A.L. (2012a). El reconocimiento de componentes y atributos del concepto de diversidad biológica en estudiantes de escuela media en Córdoba, Argentina. Memorias V Congreso Internacional de Enseñanza de la Biología. Córdoba: ADBIA, pp. 769-774.

Bermudez, G.M.A. y De Longhi, A.L. (2006). Propuesta curricular de hipótesis de progresión para conceptos estructurantes de ecología. Campo Abierto, 26 (2), pp. 13-38.

Bermudez, G.M.A. y De Longhi, A.L. (2008). La Educación Ambiental y la Ecología como ciencia. Una discusión necesaria para la enseñanza. Revista Electrónica de Educación en Ciencias, 7 (2), pp. 275-297.

Bermudez, G.M.A. y De Longhi, A.L. (2012). Análisis de la transposición didáctica del concepto de biodiversidad. Orientaciones para su enseñanza. En A. Molina, C.A. Martínez y O. Gallego (eds.). Algunas problemáticas de investigación en la enseñanza de las Ciencias Naturales en América Latina. Bogotá: Universidad Distrital Francisco José de Caldas, pp. 115-153.

Bermudez, G.M.A.; García Capocasa, M.C.; Batistón, L.V.; Díaz, S. y De Longhi, A.L. (2012b). El conocimiento de las especies vegetales nativas de estudiantes de escuela media en Córdoba, Argentina. Memorias V Congreso Internacional de Enseñanza de la Biología. Córdoba: ADBIA, pp. 833-838.

CAmpos, C.M. (2012). Los niños y la biodiversidad. ¿Qué especies conocen y cuáles son las fuentes de conocimiento sobre la biodiversidad que utilizan los estudiantes? Un aporte para definir estrategias educativas. Boletín Biológica, 24, pp. 4-9.

http://dx.doi.org/10.1016/j.jaridenv.2012.02.013

Campos, C.M.; Greco, S.; Ciarlante, J.J.; Balangione, M.; Bender, J.B.; Nates, J. y LindemannMatthies, P. (2012). Student's familiarity and initial contact with species in the Monte desert (Mendoza, Argentina). Journal of Arid Environments, 82, pp. 98-105.

Cañal, P. (2008). El nuevo currículo de biología de bachillerato: ¿qué ha cambiado y qué debe cambiar? Alambique. Didáctica de las Ciencias Experimentales, 56, pp. 35-50.

Chapin, F.S. III; Zavaleta, E.S.; Eviner, V.T.; Taylor, R.L.; Vitousek, P.M.; Reynolds, H.L.; Hooper, D.U.; Lavorel, S.; Sala, O.E.; Hobbie, S.E.; Mack, M.C. y Díaz, S. (2000). Consequences of changing biodiversity. Nature, 405, pp. 234-242.

http://dx.doi.org/10.1038/35012241

Chevallard, I. (1991). La Transposición Didáctica. Del saber sabio al saber enseñado. Buenos Aires: Aique. 
Cobo Merino, B. y Batanero, C. (2004). Significado de la media en los libros de texto de secundaria. Enseñanza de las Ciencias, 22(1), 5-18.

CBD (Convention On Biological Diversity) (2001). Secretariat of the Convention on Biological Diversity. United Nations Environment Programme. Disponible en: <http://www.biodiv.org/ convention/default.shtml\#.

Del Carmen, L.M. y Jiménez Aleixandre, M.P. (2010). Los libros de texto: un recurso flexible. Alambique Didáctica de las Ciencias Experimentales, 66, pp. 48-55.

DíAz, S. (2001a). Ecosystem function, measurement, terrestrial communities. En S. Levin (ed.). Encyclopaedia of Biodiversity. San Diego: Academic Press, vol. 2, pp. 321-344.

Díaz, S. (2001b). Does biodiversity matter to terrestrial ecosystem processes and services? En W. Steffen, J. Jäger, D. Cason y C. Bradshaw (eds.). Challenges of a changing Earth: Proceedings of the Global Change Open Science Conference. Amsterdam: Springer, pp. 165-167.

Díaz, S. y CABido, M. (2001). Vive la différence: plant functional diversity matters to ecosystem processes. TRENDS in Ecology \& Evolution, 16 (11), pp. 646-655.

http://dx.doi.org/10.1016/S0169-5347(01)02283-2

Díaz, S.; Fargione, J.; Chapin III, F.S. y Tilman, D. (2006). Biodiversity loss threatens human wellbeing. PLOS Biology, 4(8). e227.DOI:10.1371/ journal.pbio.0040277.

http://dx.doi.org/10.1371/journal.pbio.0040277

Díaz, S.; Lavorel, S.; De Bello, F.; Quétier, F.; Grigulis, K. y Robson, M. (2007). Incorporating plant functional diversity effects in ecosystem service assessments. Proceedings National Academy of Sciences USA, 104, pp. 20684-20689. http://dx.doi.org/10.1073/pnas.0704716104

Gándara Gómez, M.; Gil Quílez, M.J. y Sanmartí Puig, N. (2002). Del modelo científico de «adaptación Biológica» al modelo de «adaptación Biológica» en los libros de texto de enseñanza secundaria obligatoria. Enseñanza de las Ciencias, 20, pp. 303-314.

García, J.E. (1997). Una hipótesis de progresión sobre los modelos de desarrollo en educación ambiental. Investigación en la Escuela, 37, pp. 15-32.

García, J.E. (2003). Investigando el ecosistema. Investigación en la Escuela, 51, pp. 83-100.

García Gómez, J. y Martínez Bernat, F.J. (2010). Cómo y qué enseñar de la biodiversidad en la alfabetización científica. Enseñanza de las Ciencias, 28 (2), pp. 175-184.

Gavidia Catalán, V. y Cristerna, M.D. (2000). Dimensión medioambiental de la ecología en los libros de texto de la educación secundaria obligatoria española. Didáctica de las Ciencias Experimentales $y$ Sociales, 14, pp. 53-68.

Ghilarov, A.M. (1996). What does biodiversity means-scientific problem or convenient myth? TRENDS in Ecology and Evolution, 11 (7), pp. 304-306. http://dx.doi.org/10.1016/0169-5347(96)20016-3

Giordan, A. y De Vecchi, G. (1988). Los orígenes del saber. De las concepciones personales a los conceptos cientificos. Sevilla: Diada.

González Rodríguez, C.; García Barros, S. y Martínez losada, C. (2003). ¿'A qué contenidos relacionados con la fotosíntesis dan más importancia los textos escolares de secundaria? Enseñanza de las Ciencias, número extra, pp. 77-88.

Grime, J.P. (1997). Biodiversity and ecosystem function: the debate deepens. Science, 277, pp. 12601261.

http://dx.doi.org/10.1126/science.277.5330.1260

Hamilton, A. (2005). Species diversity or biodiversity? Journal of Environmental Management, 75, pp. 89-92.

http://dx.doi.org/10.1016/j.jenvman.2004.11.012 
Ibarra Murillo, J. y Gil Quílez, M.J. (2005). Enseñar los cambios ecológicos en la secundaria: un reto en la transposición didáctica. Enseñanza de las Ciencias, 23, pp. 345-356.

Lindemann-Matthies, P. (2005). «Loveable» mammals and «lifeless» plants: how children's interest in common local organisms can be enhanced through observation of nature. International Journal of Science Education, 27, pp. 655-677.

http://dx.doi.org/10.1080/09500690500038116

MEC (1992). Real Decreto 1178/1992, de 2 de octubre. Enseñanzas Mínimas del Bachillerato. BOE 253, 21 de octubre de 1992.

MEC (2006). Real Decreto 1631/2006, de 29 de diciembre. Educación Secundaria Obligatoria (ESO). BOE 5, viernes 5 de Enero de 2007.

MEC (2007). Real Decreto 1467/2007, de 2 de noviembre. Biología y Geología, Bachillerato LOE. BOE 266, martes 6 de noviembre de 2007.

MEC (2008). Real Decreto 67/2008, de 19 de junio. Biología, Bachillerato LOE. B.O.C.M. 152, viernes 27 de junio de 2008, pp. 6-84.

Menzel, S. y Bögeholz, S. (2009). The loss of biodiversity as a challenge for sustainable development: how do pupils in Chile and Germany perceive dilemmas? Research in Science Education, 39, pp. 429-447.

http://dx.doi.org/10.1007/s11165-008-9087-8

Munson, B.H. (1994). Ecological misconceptions. Journal of Environmental Education, 24, pp. 3034.

http://dx.doi.org/10.1080/00958964.1994.9941962

Nates, J., Campos, C.M. y Lindemann-Matthies, P. (2010). Students' perception of plant and animal species: a case study from rural Argentina. Applied Environmental Education \& Communication, 9 (2), pp. 131-141.

http://dx.doi.org/10.1080/1533015X.2010.482495

ÖZKan, Ö.; TekKaYA, C. y Geban, Ö. (2004). Facilitating conceptual change in students' understanding of ecological concepts. Journal of Science Education and Technology, 13 (1), pp. 95-105. http://dx.doi.org/10.1023/B:JOST.0000019642.15673.a3

Petchey, O.L. y Gaston, K.J. (2006). Functional diversity: back to basics and looking forward. Ecology Letters, 9, pp. 741-758.

http://dx.doi.org/10.1111/j.1461-0248.2006.00924.x

Pozo, J.I. y Gómez Crespo, M.A. (1998). Aprender y enseñar ciencias: Del conocimiento cotidiano al conocimiento cientifico. Madrid: Morata.

RoHDe, G.M. (1996). Epistemología ambiental: uma abordagem filosófica cientifica sobre la efetuacao humana alopoiética. Porto Alegre: EDIPUCRS.

Sánchez Cañete, F. y Pontes Pedrajas, A. (2010). La comprensión de conceptos en ecología y sus implicaciones para la educación ambiental. Revista Eureka sobre Enseñanza y Divulgación de las Ciencias, 7, pp. 271-285.

Snaddon, J.L., Turner, E.C. y Foster, W.A. (2008). Children's Perceptions of Rainforest Biodiversity: Which Animals Have the Lion's Share of Environmental Awareness? PLoS ONE, 3 (7). e2579. doi:10.1371/journal.pone.0002579 http://dx.doi.org/10.1371/journal.pone.0002579

Tilman, D. (2001). Functional diversity. En S.A. Levin (ed.). Encyclopedia of Biodiversity. San Diego: Academic Press, vol. 3, pp. 109-120. 


\section{ANEXO}

A) Primer año de la ESO

Fernández Esteban, M. Á., et al. 2008. Bioterra. Ciències de la naturalesa. Comunitat Valenciana. 1. Valencia: Vincens Vives Educació Secundària. 217 p. E1A.

2. Garcia Gregorio, M., et al. 1996. Ciencias de la naturaleza. Proyecto Avisor. 1.Paterna: Ecir. 209 p. E1B

3. López Fenoy, V., et al. 1996. Ciencias de la Naturaleza. 1. Zaragoza: Edelvives. 199 p. E1C.

4. Pedrinaci, E. L. C., et al. 2003. Ciencias de la naturaleza. Explora. 1. Madrid: Ediciones SM. 223 p. E1D. Bedrinaci, E. L. C., et al. 2003 . Ciencias de la naturaleza. Explora. 1. Madrid: Edicie

Balibrea, S., et al. 2007. Ciencies de la Natura. 1. Valencia: ANAYA. 255 p. E1E.

6arrio Gomez de Aguero, J., et al. 2007. Ciencies de la naturalesa. 1. Madrid: Oxford Educación. 215 p. E1F.

7. Casas Úbeda, J. M., et al. 2004. Ciencias de la Naturaleza. Serie Supernova. 1. Barcelona: Voramar Santillana. 271 p. E1G.

8. Carrión, F., et al. 1996. Ciencias de la Naturaleza. Serie Supernova. 1. Barcelona: Anaya. 167 p. E1H.

9. Brincones, I., et al. 1996. Ciències de la Naturalesa. 1. Valencia: Voramar Santillana. 174 p. E11

10. Barrio Gómez de Agüero, J., et al. 2002. Ciències de la naturalesa. Comunitat Valenciana. 1. Madrid: Oxford Educación. 239 p. E1J

11. del Carmen, L., et al. 1997. Ciències de la natura. Explora. 1. Toledo: Ediciones SM. 239 p. E1K

12. Panadero Cuartero, J. E., et al. 1996. Ciencias de la Naturaleza. 1. Madrid: Bruño. 256 p. E1L.

13. Sánchez, D., et al. 2000. Ciències de la Naturalesa. Sèrie Nova XXI, Biologia i Geologia. Crèdit comú 3. 1. Barcelona: Grup Promotor Santillana. 119 p. E1M.

B) Cuarto año de la ESO

1. Garcia, C., Riaza Moya, C. 1995. Biologia y Geologia. Ciencias de la Naturaleza. 4. Madrid: Bruño. 223 p. E4A

2. Elizalde Gómez, J. R., Sánchez Delgado, I. 1995. Biología y Geologia 4. Ciencias de la Naturaleza. Madrid: McGraw-Hill. 172 p. E4B.

3. Flores Sarrión, M. J., Gomez Gomez, S. 1998. Biología y Geología. 4. Madrid: Akal Ediciones. 307 p. E4C.

4. Balibrea, S., et al. 2003. Biología y Geologia. Ciencias de la Naturaleza. Comunidad Valenciana. 4. Barcelona: Anaya. 231 p. E4D

5. Albaladejo Marcet, C., et al. 2003. Biología y Geología. Ciencias de la Naturaleza. 4. Estella: Oxford. 239 p. E4E.

6. Berges, T., et al. 1998. Biologia i Geologia. Ciències de la Natura. Sèrie el nostre món. 4. Madrid: Anaya. 239 p. E4F.

7. Garcia Gregorio, M., et al. 2003. Biologia $i$ Geologia. ESO 4. Paterna: ECIR. 257 p. E4G.

8. Panadero Cuartero, J. E., et al. 1999. Biologia $i$ Geologia. Ciències de la Natura. Educació Secundària Obligatòria. 4. Madrid. Bruño. 223 p. E4H. Bruño. 223 p. E4H.

. Belart Rodriguez, A., et al. 1998. Biologia y Geología. Ciencias de la Naturaleza. Educación Secundaria Obligatoria. 4. Madrid. EDITEX. 205 p. E4

10. Garcia Gregorio, M., et al. 2008. Biología y Geologia. 4 ESO. Paterna: ECIR. 181 p. E4J

11. Brusi, D., et al. 2003. Biología y Geologia. 4 ESO. Comunidad Valenciana. Barcelona: Voramar Santillana. 175 p. E4K

12. Garcia Gregorio, M., et al. 1999. Biologia y Geologia. Proyecto Avizor. Educación Secundaria Obligatoria. 4. Paterna. ECIR. 170 p. E4L.

13. Garcia Giménez, L., et al. 1999. Biologia-Geologia. La evolución de la vida en un planeta cambiante. 4. Valencia: Ediciones Tilde. E4M

14. Pedrinaci, E., Gil, C. 2003. Biologia y Geología. Proyecto Ecosfera. 4. Madrid: SM. 271 p. E4N.

C) Primer año de Bachillerato:

1. Pulido, C., et al. 2002. Biología y Geologia. Bachillerato, Ciencias de la Naturaleza y de la Salud. 1. Madrid: Anaya. 342 p. B1A.

2. Garcia Gregorio, M., et al. 2003. Biología y Geología. Bachillerato. 1. Comunidad Valenciana. Valencia: ECIR. 483 p. B1B.

3. Pulido, C., et al. 2000. Biologia i Geologia. Batxillerat. Comunidad Valenciana. 1. Madrid: Anaya. 376 p. B1C

4. Ferrer Mari, N.,et al. 2008. Biologia y Geologia. Bachillerato. Madrid: Anaya. 384 p. B1D.

5. Furió Egea, J., et al. 1997. Biologia y Geología. Bachillerato. Comunidad Valenciana. Valencia: ECIR. 362 p. B1E

6. Escarré Esteve, A., et al. 1996. Biología y Geología. Bachillerato. 1. Ciencias de la Naturaleza y de la Salud. Madrid: Santillana 383 p. B1AF

7. Cabrerizo Ribate, B., et al. 2002. Biología y Geología. Bachillerato. 1. Ciencias de la Naturaleza y de la Salud. Madrid: Oxford University Press España. 371 p. B1G

8. Jimeno, A., et al. 2002. Biologia y Geologia. Bachillerato. 1. Madrid: Santillana. 399 p. B1H.

9. Anguita, F., et al. 1997. Biologia y Geologia. Bachillerato. Ciencias de la Naturaleza y de la Salud. 1. Madrid: Ediciones SM. 351 p. B11.

10. Anglada Esquius, M., et al. 1998. Biologia y Geologia I. Bachillerato. Barcelona: Edebé. 368 p. B1J.

11. Barutell Encina, A., et al. 1988. Ciencias Naturales. Bachillerato. 1. Barcelona: Anaya. 351 p. B1K

12. Arias, M., et al. 1997. Biologia 1. Batxillerat. Modalitat Ciències. Barcelona: Castellnou. 407 p. B1L.

13. Pedrinaci, E., et al. 2002. Ciencias de la Naturaleza y de la Salud. 1. Toledo: Ediciones SM. 383 p. B1M.

D) Segundo año de Bachillerato:

1. López, T. 2003. Ciencias de la Tierra y medioambientales. Bachillerato 2. Madrid: Santillana. 407 p. B2A.

2. Asensi Marqués, J. J., et al. 1998. Ciencias de la Tierra y del Medio Ambiente. Bachillerato. Ciencias de la Naturaleza y de la Salud. 2. Valencia: ECIR. 491 p. B2B.

3. Cabrera, M. E., et al. 1996. Ciencias de la Tierra y del Medio Ambiente. $2^{\circ}$ Bachillerato. Ciencias de la Naturaleza y de la Salud. Madrid: Editex 497 p. B2C

4. Gurrera, M. A., et al. 2009. Ciencias de la Tierra y del Medio Ambiente. Bachillerato 2. Modalidad de Ciencias y Tecnología. Barcelona: Edebé. 336 p. B2D.

5. Gurrera, M. A., et al. 2003. Ciencias de la Tierra y del Medio Ambiente. Bachillerato 2. Barcelona: Edebé. 336 p. B2E

6. Alfonso Cervel, F., et al. 2006. Ciencias de la Tierra y Medioambientales. $2^{\circ}$ Bachillerato. Nueva Exedra. Estella: Oxford Educación. 407 p. B2F.

Garcia Gregorio, M., et al. 2004. Ciencias de la Tierra y del Medio Ambiente. Bachillerato 2. Ciencias de la Naturaleza y de la Salud. Paterna: ECIR. 515 p. B2G

8. Rubio Sáez, N., et al. 2003. Ciencias de la Tierra y del Medio Ambiente. Bachillerato 2: Sabadell: ANAYA. 424 p. B2H

9. Diodora Calvo, M.T., et al. 2005. Ciencias de la Tierra y Medioambientales. 2. Madrid: McGraw-Hill. 436 p. B2I.

10. Diodora Calvo, M. T., Joaquin Salvachúa. 2001. Ciències de la Terra i del medi ambient, 2 Batxillerat. Madrid: McGraw-Hill. 351 p. B2J.

11. Panadero Cuartero, J. E., et al. 2007. Biología. Bachillerato. Ciencias de la Naturaleza y de la Salud. 2. Madrid: Bruño. 447 p.

12. Jimeno, A., et al. 2009. Biología 2. Bachillerato. Proyecto La Casa del Saber. Madrid: Santillana. 401 p. B2L

13. Jimeno, A., et al. 2003. Biología 2. Bachillerato. Madrid: Santillana. 414 p. B2M

14. Alcami, J., et al. 2003. Biología 2. Ciencias de la Naturaleza y de la Salud. Bachillerato. Madrid: Editorial Sm. 383 p. B2N

15. Alcami, J., et al. 2009. Biologia 2. Batxillerat. Madrid: Sm. 383 p. B2Ñ.

16. Esteller Pérez, A., et al. 2010. Biologia. Batxillerat. Ciències i Tecnologia. 2. Valencia: Vincens Vives. 401 p. B2O

17. Pulido, C. 2003. Biología. Bachillerato. Proyecto La Casa del Saber. 2. Toledo: Anaya. 448 p. B2P.

18. Miguel González, C. A., et al. 2003. Biología. Bachillerato Everest. 2. León: Everest. 398 p. B2Q.

19. Granados Martinez, F., López Fenoy, V. 2003. Biología. Ciencias de la Naturaleza y Salud. 2. Zaragoza: Edelvives. 359 p. B2R

20. Garcia Gregorio, M., et al. 2003. Biologia. Batxillerat. 2. Paterna: Ecir. 403. B2S 


\section{Didactic transposition of the biodiversity concept. A study of Spanish high-school textbooks}

\author{
Gonzalo M.A. Bermudez \\ Universidad Nacional de Córdoba, Facultad de Ciencias Exactas, \\ Físicas y Naturales, Argentina. - CONICET (Consejo Nacional de \\ Investigaciones Científicas y Técnicas), Argentina. - Universidad de \\ Valencia, Facultad de Magisterio, Departamento de Didáctica de las \\ Ciencias Experimentales y Sociales \\ gbermudez@com.uncor.edu \\ Sandra Díaz \\ Universidad Nacional de Córdoba, Facultad de Ciencias Exactas, Físi- \\ cas y Naturales, Argentina. • CONICET (Consejo Nacional de Inves- \\ tigaciones Científicas y Técnicas), Argentina.
}

\author{
Ana Lía De Longhi \\ Universidad Nacional De Córdoba, Facultad de \\ Ciencias Exactas, Físicas y Naturales, Argentina.
}

This paper examines the existence and complexity of the treatment of the biodiversity concept in middle and high school Spanish textbooks. There is a consensus that science teaching should provide students with a theoretical foundation and a practical guide to support decision-making skills related to biodiversity conservation. However, many textbooks treat the concept of biodiversity as being decontextualised from the social sphere, even though the ethical and political dimensions are substantial.

This was initially studied in the framework of mathematics education by Chevallard who stated that the route of reference knowledge ("scholarly" or "original" knowledge) up to the moment when it is implemented in the classroom ("knowledge to be taught" and that is effectively "taught") requires a great deal of transpositive work (or "didactic transposition"), whereas the construction of new knowledge arises with epistemological and functional statuses that differ from the reference knowledge. The topics to be taught then take on a new shape, accompanied by a process of de-personalising, de-syncretising, de-contextualising, publicity and programmability to be re-contextualized into a new discourse in textbooks and teaching materials. In this context, the main objectives of this research were three-fold: (a) to develop a conceptual analysis grid of the biodiversity concept, which can be applied to textbooks of secondary education, (b) to analyse the didactic transposition of the concept of biodiversity that is promoted in high school (Bachillerato) and middle school (Educación Secundaria Obligatoria, ESO) textbooks; and (c) to establish the levels of complexity of the treatment of biodiversity in textbooks of secondary education by defining a set of variables. First, and with this purpose in mind, scientific papers from the "expert community" were reviewed in order to define the biodiversity conceptual framework; secondly, the national curriculum proposed by the Spanish Ministry of Education was analysed in order to determine the presence of the biodiversity concept. Finally, a third phase consisted of an analysis of the presence/absence of this concept in textbooks. A total of 60 textbooks from middle school ( $n=27)$ and high school level $(n=33)$ were selected (older than 1995), with the response variable being the presence or absence of this concept in each category's specified content analysis.

After carrying out an analysis of "expert" definitions of biodiversity occurring in scientific papers and encyclopaedias, it was found that conceptualisation has become wider over recent decades. After syllabi analysis, the biodiversity concept was observed to be present in the first and the fourth grades of middle school in the "Natural Sciences" and "Biology and Geology" subjects, respectively, as well as in the first and second grades in high school in the "Biology I" and "Earth and Environmental Sciences" subjects, respectively.

The conceptualisation of biodiversity occurred in most of the textbooks analysed, with "species" being the most prominent component, while other components such as genetics/population, community/ecosystem, landscape and functional groups were absent in the vast majority of textbooks. These findings reveal that the transpositive process for conceptualising biodiversity is outdated with respect to the definitions given in the scientific community. This phenomenon, denoted by Chevallard as "biological aging", occurs when the gap between the scientific community's knowledge and the "knowledge to be taught" increases. Regarding biodiversity attributes, "richness" was the most frequently occurring one in the manuals of both educational levels, although the definition of attributes such as "species richness" and "relative abundance" were more complex in the second year of high school, with estimates of species diversity indices being proposed. To study the complexity levels, a Main Component Analysis was performed, and it was found that most of the textbooks for the second year in high school represented the highest level in the conceptual treatment of biodiversity, while the lowest level was largely composed of textbooks for the first year of middle school. Minor changes from the first to the fourth year in middle school were also observed. Taking into account all the above findings, we recommend the use of the 'Earth and Environmental Sciences' textbooks to transpose the biodiversity concept for all grades in secondary education. Additionally, the intricate process of transposition revealed that the official national curriculum does not guarantee a conceptualisation of biodiversity more in line with that of the "scientific community". 